\title{
A Single Nucleotide Polymorphism Fine Mapping Study of Chromosome 1q42.1 Reveals the Vulnerability Genes for Schizophrenia, GNPAT and DISC1: Association with Impairment of Sustained Attention
}

\author{
Yu-Li Liu, Cathy Shen-Jang Fann, Chih-Min Liu, Wei J. Chen, Jer-Yuarn Wu, Shuen-lu Hung, \\ Chun-Houh Chen, Yuh-Shan Jou, Shih-Kai Liu, Tzung-Jeng Hwang, Ming H. Hsieh, Wen-Chen Ouyang, \\ Hung-Yu Chan, Jiann-Jyh Chen, Wei-Chih Yang, Chin-Yu Lin, Sandy F.C. Lee, and Hai-Gwo Hwu
}

\begin{abstract}
Background: The marker D1S251 of chromosome 1q42.1 showed significant association with schizophrenia in a Taiwanese sample. We used single nucleotide polymorphism (SNP) fine mapping to search for the vulnerability genes of schizophrenia.

Methods: We selected 120 SNPS covering 1 Mb around D1S251 from the public database. These selected SNPs were initially validated if allele frequency was $>10 \%$. Forty-seven validated SNPs were genotyped in 102 families with at least 2 siblings affected with schizophrenia.

Results: Two SNP blocks showed significant association with schizophrenia. Block 1 (five-SNP), located between intron 2 and intron 13 of the glyceronephosphate O-acyltransferase (GNPAT) gene, showed the most significant associations using single-locus TDT (z = $-2.07, \mathrm{p}=.038, \mathrm{df}=1)$ and haplotype association analyses $(\mathrm{z}=-1.99, \mathrm{p}=.046, \mathrm{df}=1)$. Block. $2($ two-SNP), located between intron 4 and intron 5 of the disrupted-in-schizophrenia 1 (DISC1) gene, also showed the most significant results in both the single-locus $(\mathrm{z}=-3.22, \mathrm{p}=.0013, \mathrm{df}=1)$ and haplotype association analyses $(\mathrm{z}=3.35, \mathrm{p}=.0008, \mathrm{df}=1)$. The association of the DISC1 gene with schizophrenia was mainly in the patient group with sustained attention deficits as assessed by the Continuous Performance Test. Conclusions: Chromosome 1q42.1 harbors GNPAT and DISC1 as candidate genes for schizophrenia, and DISC1 is associated with sustained attention deficits.
\end{abstract}

$\overline{\text { Key Words: Schizophrenia, sustained attention, DISC1, GNPAT, hap- }}$ lotype association, quantitative TDT

S chizophrenia is a complex genetic disorder with several genes in epistasis for its etiology (Risch 1990). One promising chromosome region, possibly hosting the candidate vulnerability genes of schizophrenia, is 1q 42, where a balanced translocation $(1 ; 11)(\mathrm{q} 42.1 ; \mathrm{q} 14.3)$ disrupted two genes (i.e., disrupted-inschizophrenia 1 [DISC1] and DISC2); this region has been found to be associated with major mental illnesses including schizophrenia in a large Scottish family pedigree (Blackwood et al 2001; Millar et al 2000; Millar et al 2001). This region of linkage evidence has also been independently confirmed in a Finnish family sample (Hovatta et al 1999) a North American white population

From the Division of Mental Health and Substance Abuse Research (Y-LL), National Health Research Institutes, Taipei; the Institute of Biomedical Sciences (CS-JF, Y-SJ, WCY, C-YL), Academia Sinica, Taipei; the Department of Psychiatry (C-ML, WJC, S-KL, T-JH, MHH, H-GH), National Taiwan University Hospital; the Institute of Epidemiology (WJC, H-GH), College of Public Health, National Taiwan University, Taipei; the National Genotyping Center (J-YW, S-IH), the Institute of Biomedical Sciences, Academia Sinica, Taipei; the Institute of Statistical Science (C-HC), Academia Sinica, Taipei; the National Chia-Nan Psychiatric Center (W-CO), Tainan; the National Taoyuan Psychiatric Center (H-YC, J-JC, SFCL), Taoyuan; and the Department of Psychology (H-GH), College of Science, National Taiwan University, Taipei, Taiwan.

Address reprint requests to Hai-Gwo Hwu, M.D., Department of Psychiatry, National Taiwan University Hospital, No. 7, Chung Shan South Road, Taipei, Taiwan, ROC 100; E-mail: haigohwu@ha.mc.ntu.edu.tw.

Received November 30, 2005; revised April 21, 2006; accepted April 25, 2006.
(Hodgkinson et al 2004), and a Taiwan family sample (Hwu et al 2003). There is even evidence showing that the variations in DISC1 may affect hippocampal structure and function (Callicott et al 2005). However, the linkage evidence has not been consistently supported in other ethnic groups (Bassett et al 2002; Levinson et al 2002; Macgregor et al 2002).

Because of the concern about the heterogeneity and inconsistent findings regarding linkage studies of schizophrenia, using certain endophenotype to refine the phenotype characterization has been advocated (Gottesman and Gould 2003). Both sustained attention deficit and executive dysfunction have substantial empirical evidence to support them as potential candidates for such endophenotypic markers. First, sustained attention deficits as measured on the Continuous Performance Test (CPT) (Rosvold et al 1956) have been shown to be presented not only in schizophrenic patients, but also in subjects with schizotypal personality disorder and in nonpsychotic relatives of schizophrenic patients (Cornblatt and Keilp 1994; Chen and Faraone 2000). Using SDs of 2.5 or more below the population mean as the threshold, we found that the recurrence risk ratio for CPT performance among parents or siblings was higher than that of schizophrenia alone (Chen et al 1998b; Chen et al 2004). Second, executive functions as measured by the Wisconsin Card Sorting Test (WCST) (Robinson et al 1980) are known to be impaired in schizophrenic patients (Goldberg et al 1987; Koren et al 1998) and their first degree relatives (Wolf et al 2002). Among schizophrenic patients, impaired executive functioning has been related to hypofrontality (Weinberger et al 1988).

Using both the CPT and WCST to define endophenotypes for schizophrenia might be helpful in addressing the heterogeneity and variable expression of schizophrenia in linkage analyses. On the basis of a previous finding that the short tandem repeat 
marker D1S251 located within the gap between the TRAX and DISC1 genes had significant linkage with schizophrenia in a Taiwanese sample (Hwu et al 2003), we aimed in this study to pursue the possible significant association of SNP markers located near the D1S251 region using the SNP fine mapping. Our first hypothesis was that if there are some SNP markers near the D1S251 marker found to be significantly associated with schizophrenia; these SNP markers would belong to a significant haplotype located in some functional genes expressed in the brain. In addition to the clinical diagnosis of schizophrenia, we intended to examine the relations between the CPT and WCST performance and the SNPs. If there is an association between schizophrenia and some SNPs, our second hypothesis was that the association would become more significant in the subgroup of patients with a certain endophenotype but become nonsignificant in the subgroup without the endophenotype.

\section{Methods and Materials}

\section{Subjects}

Schizophrenic patients who had at least one affected sibling (the proband cases) were identified from the Department of Psychiatry, National Taiwan University Hospital, and the university-affiliated Taoyuan Psychiatric Center. This research project was approved by the Institutional Review Board of National Taiwan University Hospital. Data collection was initiated after informed consents were obtained from the identified study subjects and their families. All family members were personally interviewed by the research psychiatrists with the use of the Psychiatrist Diagnostic Assessment (PDA) (Hwu 1999). The final diagnostic assessment was formulated by integrating the PDA data and clinical information of medical chart records. The final diagnosis was done following the DSM-IV criteria for schizophrenia, schizoaffective disorder, and other nonaffective psychoses. Clinical data of age at onset of initial symptoms, negative symptoms, and positive symptoms were collected. The negative and positive symptoms were assessed with the use of the schedule for assessment of negative symptoms (SANS; Andreasen 1983) and the schedule for assessment of positive symptoms (SAPS; Andreasen 1984) with satisfactory reliability. A negative symptom score was the sum of all global scores of five negative symptom dimensions, including affective flattening, alogia, avolition/apathy, anhedonia/asociality, and impaired attention. Positive symptom score was the sum of all global scores of four positive symptom dimensions, including hallucination, delusion, excitements, and thought derailment.

In total, 102 schizophrenic nuclear families with at least two affected siblings were recruited for this study. Among the 399 individuals, 231 individuals underwent an undegraded CPT test, and 225 individuals underwent a degraded CPT test. Meanwhile, 164 subjects underwent WCST assessment.

\section{Neuropsychological Assessment}

Continuous Performance Test. A CPT machine from Sunrise System version 2.20 (Pembroke, Massachusetts) was used to assess sustained attention. The procedure has been described in detail elsewhere (Chen et al 1998a). Briefly, numbers from 0 to 9 were randomly presented for $50 \mathrm{msec}$ each, at a rate of one per second. Each subject undertook two CPT sessions: the undegraded 1-9 task and the 25\% degraded 1-9 task. Subjects were asked to respond whenever the number "9" preceded by the number " 1 " appeared on the screen. A total of 331 trials, 34 (10\%) of which were target stimuli, were presented over $5 \mathrm{~min}$ for each session. During the $25 \%$ degraded session, a pattern of snow was used to toggle background and foreground so that the image was visually distorted. Each test session began with 2 min of practice (repeated if subjects required). One signal-detection index of performance on the test, sensitivity $\left(\mathrm{d}^{\prime}\right)$, was derived from the hit rate (probability of response to target trials) and false-alarm rate (probability of response to nontarget trials) (Nuechterlein 1991). Sensitivity is an individual's ability to discriminate target stimuli from nontarget stimuli. In a 1-week test-retest reliability study (Chen et al 1998a) of the CPT versions used in this study, the intraclass correlation coefficients or reliability of $\mathrm{d}^{\prime}$ were .83 and .82 for the undegraded and the $25 \%$ degraded 1-9 task, respectively.

Wisconsin Card Sorting Test. We employed a computerized version of the WCST (Tien et al 1996) that had been applied in a previous study in a Taiwanese population (Lin et al 2000). During the WCST, subjects were required to match response cards to the four stimulus cards according to one of three dimensions (color, form, or number) by pressing one of the 1 to 4 number keys on the computer keyboard. Subjects were not informed of the correct sorting principle nor were they told when the principle would shift during the test, but they were given feedback ("Right" or "Wrong") on the screen after each trial. Unlike one common form of the traditional WCST in which the test ends after six correct categories are achieved, the testing in this study continued until all 128 cards were sorted. All of the indexes defined in the WCST manual (Heaton et al 1993), except for Total Correct, were used for analysis. The Total Correct index was not included, since it is complementary to Total Errors. The indexes used were 1) Total Errors: total number of perseverative and nonperseverative errors; 2) Nonperseverative Errors: number of errors that were not perseverative; 3) Perseverative Errors: number of errors that were perseverative, reflecting a tendency toward perseveration; 4) Perseverative Responses: number of responses that were perseverative, regardless of whether they were correct or not; 5) Categories Achieved: number of times that 10 correct responses in a row were made, reflecting overall success; 6) Trials to Complete First Category: number of trials to successfully complete the first category (counted as 129 if no category was completed), reflecting initial conceptual ability; 7) Conceptual Level Response: proportion of consecutive correct responses occurring in runs of three or more, reflecting insight into the correct sorting principles; 8) Failure to Maintain Set: number of times subject makes five to nine correct responses in a row, reflecting efficiency of sorting; and 9) Learning to Learn: average difference in percent errors between successive categories, reflecting the average change in conceptual efficiency during the test (Heaton et al 1993). The last index can be calculated only for subjects whose total numbers of Categories Achieved and categories attempted are larger than three.

\section{SNP Selection Criteria and Validation}

Fine mapping studies were using SNP dense markers spreading upstream and down stream of the dinucleotide marker D1S251 located at 1q42.1. In the defined region of $2 \mathrm{cM}$ around DIS251, a total of 120 SNPs were selected covering 10 genes from public database (http://www.ensembl.org/Homo_sapiens/martview). The inter SNP marker distance ranged from $5 \mathrm{~kb}$ to $60 \mathrm{~kb}$, with an average of $32.5 \mathrm{~kb}$. According to the location of these SNPs relative to the position of the functional genes, the SNPs were selected based upon the following priority of exon (including $5^{\prime}$-untranslated and 3-untranslated regions), promoter (CpG island), intron, and gap between functional genes that are expressed in the central nervous system. The 120 SNPs selected this 
way were nearly evenly distributed, with 8 being in exons, 3 in promoters, and the rest in introns. We used 31 trios and 2 independent individuals, a total of 95 individuals, to validate the 120 SNPs.

\section{SNP Genotyping}

All SNP genotypings were performed by the method of matrixassisted laser desorption/ionization-time of flight mass spectrometry (MALDI-TOF MS) (Rodi et al 2002). Primers and probes flanking the SNPs were designed by using SpectroDESIGNER software (Sequenom, San Diego, California). A DNA fragment (100-300 bp) encompassing the SNP site was amplified by using the polymerase chain reaction (PCR) (GeneAmp 9700 thermocycler, Applied Biosystems, Foster City, California) according to the manufacturer's instruction.

After removing the unincorporated deoxynunleotide triphosphate (dNTP) and inactivating the shrimp alkaline phosphatase (SAP) from the PCR product, primer extension was performed by adding the probe, Thermo Sequenase (Amersham Pharmacia, Piscataway, New Jersey), and appropriate dideoxynucleotide triphosphate (ddNTP)/dNTP mixture, and was followed by 55 cycles of denaturing at $94^{\circ} \mathrm{C}$ for $5 \mathrm{sec}$, annealing at $52^{\circ} \mathrm{C}$ for $5 \mathrm{sec}$, and extension at $72^{\circ} \mathrm{C}$ for $5 \mathrm{sec}$. Different extension products were differentiated by mass through MALDI-TOF.

This genotyping method has been applied to a broad variety of clinical applications, since it fulfills criteria such as accuracy of SNP detection, sensitivity to score SNPs using a small amount of template throughput capacity, flexibility of the procedure, and cost-effectiveness (Tost and Gut 2005).

\section{Statistical Analysis}

To verify the sample accuracy, including family relationship and genotype, we used PEDCHECK version 1.1 (O'Connell and Weeks 1998) and UNKNOWN version 5.23 (Terwilliger and Ott 1994) to check Mendelian inheritance, and Procedure ALLELE in SAS/GENETICS release 8.2 (SAS Institute, Cary, North Carolina; SAS 2002) was used to test for Hardy-Weinberg equilibrium. Linkage disequilibrium of intermarkers was measured by using coefficient D'(Hedrick 1987), which was also used to define haplotype blocks. A graphic presentation of block pattern was completed with the use of Haploview software (Barrett et al 2005).

Family-based transmission disequilibrium tests were applied to test linkage disequilibrium. Both single-locus and haplotypebased association analyses were carried out simultaneously by using two popular programs for the nuclear family data, haplotype FBAT version 1.4.1 for affected offspring association analyses (Horvath et al 2001; Horvath et al 2004; Laird et al 2000) and TRANSMIT version 2.5.4 for parent to affected offspring association analyses (Clayton 1999). An individual's haplotype was inferred with the use of SimWalk2 version 2.86 (Sobel and Lange 1996; Sobel et al 2001; Sobel et al 2002), which uses the Markov Chain Monte Carlo algorithm. Moreover, the Generalized Estimating Equations (GEE) method (Liang and Zeger 1986) was applied to test the interaction between haplotype blocks with the use of the Proc GENMODE of SAS version 8.0 for Windows (SAS Institute, Cary, North Carolina).

Multiple tests were considered to be necessary. However, the SNP markers used in this study were high density and the application of the Bonferroni procedure might yield too conservative results. In this study, we applied a simulation study, using Merlin software (Abecasis et al 2002) to simulate the pedigree for 5000 times assuming no linkage/no association on the interested SNP marker identified in the TRANSMIT and FBAT programs. The empirical $p$ values $(\alpha=.05)$ for these identified SNP markers of interest were considered equivalent to the results of multiple testing.

Besides the analysis of qualitative trait, quantitative analysis using highly heritable quantitative trait was also considered. The analysis of heritability and quantitative-type transmission disequilibrium test based on variance component approach was applied with the use of QTDT version 2.4.3 (Abecasis et al 2000a; Abecasis et al 2000b).

\section{Results}

\section{SNP Validation}

An SNP was considered valid if the frequency of minor allele was larger than 10\% and the genotyping missing rate was smaller than 30\%. Forty-seven of 120 SNPs met the validity criteria. The 47 SNPs span across $1591 \mathrm{~kb}$ around the D1S251 marker (Table 1) and cover 12 known functional genes of COG2, AGT, CAPN9, FLJ14525, FLJ 22584, ARV1, TRIM67, GNPAT, DKFZP547NO43 (Clorf124), EGLN1, TRAX, and DISC1. Four genes, CAPN9, ARV1, TRIM67, and Clorf124, did not have valid SNPs for further analyses. As only founders were included in the Hardy-Weinberg equilibrium test, five SNPs (SNP495, 506, 513, 527, 581) that violate the test were excluded from further analysis.

\section{Construction of SNP Block}

To perform haplotype analysis, we evaluated haplotype blocks using intermarker linkage disequilibrium coefficient. Two SNP blocks were identified by using two criteria: 1) a significant intermarker association based on the chi-square test, and 2) coefficient $\mathrm{D}^{\prime}$ is higher than .8. The locations of these two SNP blocks are shown in Figure 1. The first block covers the SNP markers of 482 (intron 2), 485 (intron 2), 479 (intron 5), 488 (intron 11), and 489 (intron 13) in the GNPAT gene region, and the second block covers the markers of 517 (intron 4) and 518 (intron 5) in the DISC1 gene region (Figure 2).

\section{Single-Locus Association Analysis}

Preliminary analyses were conducted in nuclear families to evaluate the potential association between each SNP and phenotype, defined in either a narrow model (DSM-IV schizophrenia only) or broad model (DSM-IV schizophrenia, schizoaffective disorder, and other nonaffective psychotic disorders). From the results of single-locus association analyses using the computer program FBAT, which is robust for population admixtures, we found that some SNP variants exhibited a significant association with schizophrenia (Table 2). For the broad model, the significant SNP marker on the GNPAT gene was SNP 485 (rs508908) $(p=.0383)$, and those on the DISC1 gene were SNP517 $(\mathrm{rs} 2793092)(p=.0053)$ and SNP518 $(\mathrm{rs} 2793091)(p=.0076)$. For the narrow model, the SNP marker 485 (rs508908) ( $p=.0701)$ on the GNPAT gene showed a borderline effect, whereas SNP 517 $(\mathrm{rs} 2793092)(p=.0013)$ and $518(\mathrm{rs} 2793091)(p=.0071)$ on the DISC1 gene exhibited highly significant associations. For comparison, similar analyses using the computer program TRANSMIT, which can utilize data from all families, even when parental genotypes are unknown, yielded similar results but with more significance, especially for the SNPs on the GNPAT gene under the narrow model of phenotype (i.e., 485 [rs508908] [ $p=.019$ ] and 479 [rs538643] [ $p=.049])$.

These three identified SNP markers, rs508908 (GNPAT, primer id 485), rs2793092 (DISC1, primer id 517), and rs2793091 (DISC1, primer id 518), were under simulation for 5000 times assuming no linkage and no association. For these simulated pedigrees, FBAT was used to calculate $p$ values. The simulated results have 
Table 1. Description of 47 SNP Markers

\begin{tabular}{|c|c|c|c|c|c|c|}
\hline $\begin{array}{l}\text { Gene or } \\
\text { Gap (G) }\end{array}$ & $\begin{array}{l}\text { SNP Study } \\
\text { Number }\end{array}$ & SNP ID & $\begin{array}{c}\text { No. of } \\
\text { Families }\end{array}$ & Allele Type & $\begin{array}{l}\text { Minor Allele } \\
\text { Frequency }\end{array}$ & $\begin{array}{l}\text { Chromosomal } \\
\text { Position }\end{array}$ \\
\hline G & 565 & rs917384 & 101 & $\mathrm{G} / \mathrm{C}$ & .5652 & chr1:227185526 \\
\hline COG2 & 472 & rs1887492 & 101 & $\mathrm{G} / \mathrm{A}$ & .1585 & chr1:227252942 \\
\hline AGT & 471 & rs699 & 102 & $\mathrm{G} / \mathrm{A}$ & .2000 & chr1:227318982 \\
\hline FLJ14525 & 478 & rs1202566 & 100 & $\mathrm{G} / \mathrm{A}$ & .3648 & chr1:227461322 \\
\hline FLJ22584 & 484 & rs2275333 & 101 & $\mathrm{~A} / \mathrm{T}$ & .4024 & chr1:227530435 \\
\hline FLJ22584 & 475 & rs2153051 & 101 & $\mathrm{~T} / \mathrm{C}$ & .4187 & chr1:227549768 \\
\hline G & 576 & rs2024816 & 98 & $\mathrm{C} / \mathrm{T}$ & .1250 & chr1:227707934 \\
\hline G & 569 & rs765265 & 91 & $\mathrm{G} / \mathrm{A}$ & .385 & chr1:227749195 \\
\hline GNPAT & 482 & rs487047 & 101 & $\mathrm{G} / \mathrm{A}$ & .3871 & chr1:227850811 \\
\hline GNPAT & 485 & rs508908 & 100 & $\mathrm{~A} / \mathrm{T}$ & .3843 & chr1:227859800 \\
\hline GNPAT & 479 & rs538643 & 102 & $\mathrm{~T} / \mathrm{C}$ & .388 & chr1:227873448 \\
\hline GNPAT & 488 & rs539699 & 102 & $\mathrm{~T} / \mathrm{C}$ & .384 & chr1:227880149 \\
\hline GNPAT & 489 & rs578945 & 102 & $\mathrm{~T} / \mathrm{C}$ & .388 & chr1:227883303 \\
\hline EGLN1 & 483 & rs1435167 & 102 & $\mathrm{~A} / \mathrm{T}$ & .468 & chr1:227975433 \\
\hline TSNAX & 494 & rs1621135 & 102 & $\mathrm{~A} / \mathrm{T}$ & .504 & chr1:228137941 \\
\hline TSNAX & 501 & rs1655290 & 102 & $\mathrm{C} / \mathrm{T}$ & .504 & chr1:228143015 \\
\hline TSNAX & 491 & rs1615409 & 101 & $\mathrm{~A} / \mathrm{C}$ & .4797 & chr1:228156605 \\
\hline TSNAX & 496 & rs766288 & 101 & $\mathrm{G} / \mathrm{T}$ & .3252 & chr1:228166876 \\
\hline G & 581 & rs892356 & 102 & $\mathrm{C} / \mathrm{T}$ & .2114 & chr1:228220138 \\
\hline DISC1 & 6055 & rs1030711 & 85 & $\mathrm{~A} / \mathrm{T}$ & .1316 & chr1:228228649 \\
\hline DISC1 & 6054 & rs1865226 & 94 & $\mathrm{C} / \mathrm{T}$ & .2714 & chr1:228231070 \\
\hline DISC1 & 502 & rs1865225 & 101 & $\mathrm{~A} / \mathrm{G}$ & .4065 & chr1:228236192 \\
\hline DISC1 & 498 & rs2082552 & 101 & $\mathrm{~T} / \mathrm{C}$ & .4836 & chr1:228241078 \\
\hline DISC1 & 495 & rs1094658 & 102 & $\mathrm{C} / \mathrm{G}$ & .18 & chr1:228246585 \\
\hline DISC1 & 499 & rs980394 & 102 & $A / G$ & .184 & chr1:228252695 \\
\hline DISC1 & 506 & rs1417585 & 79 & $\mathrm{~T} / \mathrm{C}$ & .1822 & chr1:228276792 \\
\hline DISC1 & 513 & rs1417584 & 100 & $\mathrm{C} / \mathrm{T}$ & .1736 & chr1:228292238 \\
\hline DISC1 & 524 & rs1977797 & 92 & $\mathrm{~T} / \mathrm{A}$ & .4363 & chr1:228298262 \\
\hline DISC1 & 514 & rs1954175 & 92 & $\mathrm{~T} / \mathrm{C}$ & .4451 & chr1:228328598 \\
\hline DISC1 & 517 & rs2793092 & 102 & $\mathrm{~A} / \mathrm{G}$ & .449 & chr1:228353528 \\
\hline DISC1 & 518 & rs2793091 & 101 & $\mathrm{G} / \mathrm{A}$ & .4569 & chr1:228367796 \\
\hline DISC1 & 590 & rs2812393 & 100 & $\mathrm{C} / \mathrm{G}$ & .3941 & chr1:228386861 \\
\hline DISC1 & 526 & rs1407598 & 99 & $\mathrm{~T} / \mathrm{G}$ & .3621 & chr1:228420013 \\
\hline DISC1 & 529 & rs1000730 & 85 & $\mathrm{C} / \mathrm{T}$ & .396 & chr1:228436789 \\
\hline DISC1 & 527 & rs734551 & 85 & $\mathrm{G} / \mathrm{A}$ & .4624 & chr1:228467621 \\
\hline DISC1 & 531 & rs999710 & 89 & $A / G$ & .4485 & chr1:228484131 \\
\hline DISC1 & 544 & rs999709 & 100 & $\mathrm{~A} / \mathrm{G}$ & .4487 & chr1:228484218 \\
\hline DISC1 & 535 & rs967433 & 101 & $\mathrm{~T} / \mathrm{C}$ & .3554 & chr1:228505003 \\
\hline DISC1 & 541 & rs2038636 & 101 & $\mathrm{G} / \mathrm{A}$ & .1992 & chr1:228528437 \\
\hline DISC1 & 548 & rs928100 & 102 & $\mathrm{G} / \mathrm{C}$ & .3525 & chr1:228543084 \\
\hline DISC1 & 543 & rs1417866 & 91 & $A / G$ & .3333 & chr1:228560101 \\
\hline DISC1 & 547 & rs701160 & 101 & $\mathrm{C} / \mathrm{T}$ & .2438 & chr1:228565669 \\
\hline DISC1 & 552 & rs701161 & 98 & $A / G$ & .4397 & chr1:228577302 \\
\hline DISC1 & 557 & rs821664 & 102 & $\mathrm{C} / \mathrm{T}$ & .2917 & chr1:228595110 \\
\hline DISC1 & 6056 & rs821616 & 102 & $\mathrm{~A} / \mathrm{T}$ & .127 & chr1:228617786 \\
\hline G & 582 & rs1338302 & 102 & $\mathrm{C} / \mathrm{T}$ & .2927 & chr1:228751787 \\
\hline G & 583 & rs1766982 & 101 & $A / G$ & .3571 & chr1:228847915 \\
\hline
\end{tabular}

shown that empirical $p$ values were .043, .043, and .05 for rs508908, rs2793092, and rs2793091, respectively. Thus, we considered that these results were not false positive.

\section{Haplotype-Based Association Analysis}

Since there were SNPs in each block that exhibited single-locus association with schizophrenia, either narrowly or broadly defined, haplotype-based association analysis was further pursued. The results from the haplotype FBAT program version 1.4.1 (Horvath et al 2004) showed that haplotype GATTT in the GNPAT gene SNP block (block 1) is only slightly significant for the broad model of schizophrenia phenotype ( $p=.0461$ ) (Table 2$)$. The haplotypes AG and GA in the DISC1 gene SNP block (block 2) exhibited a significant association for both the narrow model $(p=.0091$ for AG and $p=.0008$ for GA) and the broad model ( $p=.0295$ for AG and $p=.0017$ for GA) of schizophrenia. It seemed that haplotype GA was a risk haplotype (negative $Z$ statistic), whereas AG possessed a protection effect. When the haplotypes in the DISC1 gene block were examined separately for females and males, a similar pattern of overtransmission for GA and undertransmission for AG was found in each gender, although the magnitude of significance was reduced (data not shown). Analyses carried out with the TRANSMIT program yielded a similar pattern, but the results are not shown here. The interaction of two haplotype blocks was investigated by using the GEE method. However, no significant interaction effect between the two was found. 


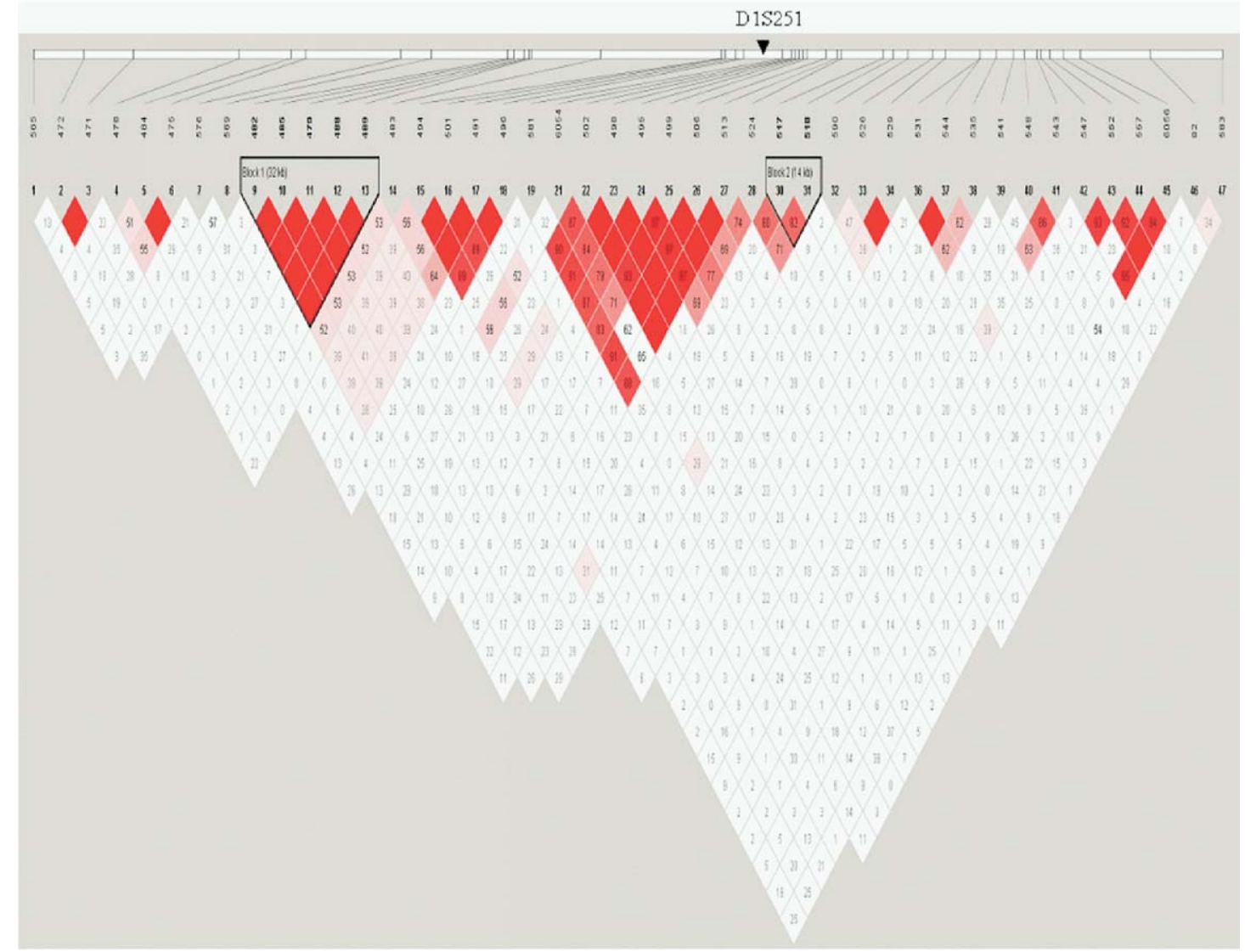

Figure 1. Linkage disequilibrium of all SNP markers showed two significant SNP blocks located within a block of $482,485,479,488$, and 489 , and a block of 517 and 518 .

\section{Quantitative TDT for Phenotypic Indicators}

In exploring the relationship between the SNP genotypes and potential endophenotypes, we examined four types of traits: the age of onset of the initial symptom, clinical symptoms (Positive and Negative Syndrome Scale [PANSS] Negative Scale scores, PANSS Positive Scale scores), CPT scores (undegraded CPT d',

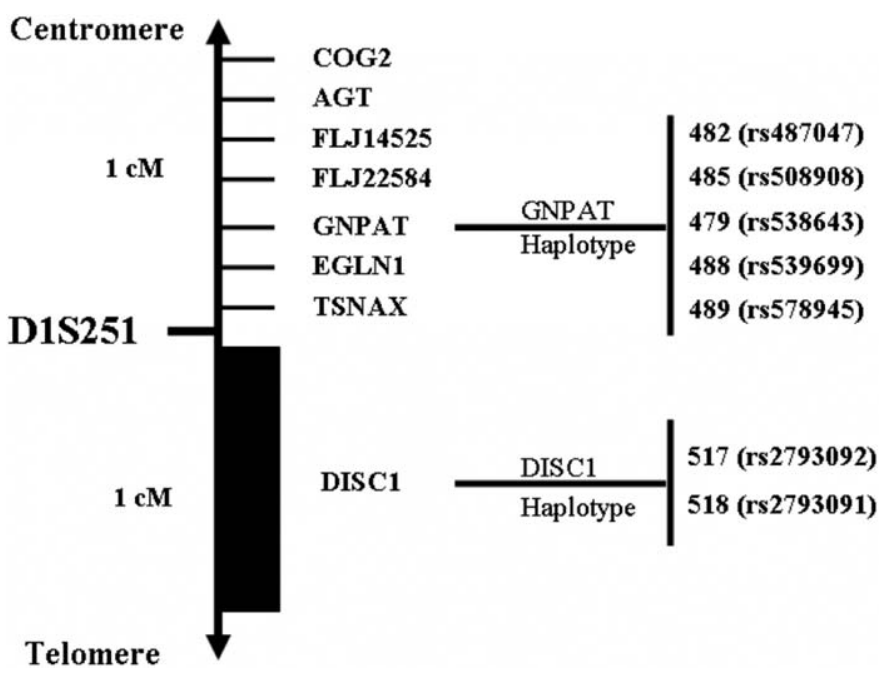

Figure 2. Blocks of SNPs located within genes of GNPAT and DISC1 showed significant linkage disequilibrium and associations among nine other functional genes in the 2-cM ranges around D1S251 marker. degraded CPT d'), and WCST scores (total errors, nonperseverative errors, perseverative errors, perseverative responses, categories achieved, conceptual level responses, trials to complete first category, learning to learn, and failure to maintain set). The heritability analysis using QTDT was conducted among the schizophrenic families to screen for important quantitative phenotypes, in which the variance components were decomposed because of environment $\left(\sigma_{\mathrm{e}}{ }^{2}\right)$ and genetic effect $\left(\sigma_{\mathrm{g}}{ }^{2}\right)$. Heritability $h^{2}$ is defined as the explained proportion of the variance attributable to genetic component (i.e., $\sigma_{\mathrm{g}}{ }^{2} /\left(\sigma_{\mathrm{g}}{ }^{2}+\sigma_{\mathrm{e}}{ }^{2}\right)$ ). Among the 14 definitions examined, only two traits were found to have a significant heritability on the basis of the likelihood ratio test: the undegraded CPT $\mathrm{d}^{\prime}\left(h^{2}=.831, p=8 \times 10^{-7}\right)$ and the degraded CPT d' $\left(b^{2}=.751, p=2 \times 10^{-7}\right)$.

A quantitative haplotype analysis that detects unbalanced allelic transmission and important variance components was then conducted by using these two highly heritable traits. Among different phenotypic mean and variance component models, we applied the Akaike information criterion (AIC) to select the optimum genetic model underlying the linkage and association with schizophrenia. Since the AIC was used only as a criterion to evaluate the fitness of model, we did not describe the significance level and did not perform the correction for multiple testing in these analyses either. Of note, the response criterion $\ln \beta$ of the CPT was regarded as a covariate to be adjusted for in the model fitting because it reflected an individual motivation for the test. The fitted models showed that the first haplotype block was linked and associated the degraded CPT d' $(\log$ likelihood $=$ 
Table 2. Haplotype Analysis of All Families Using FBAT Program

\begin{tabular}{|c|c|c|c|c|c|c|c|}
\hline & \multirow[b]{2}{*}{$\mathrm{HF}$} & \multicolumn{3}{|c|}{ Narrow Model } & \multicolumn{3}{|c|}{ Broad Model } \\
\hline & & $N^{a}$ & $Z^{b}$ & $p$ Value & $N$ & $Z$ & $p$ Value \\
\hline \multicolumn{8}{|c|}{ GNPAT (Block 1) } \\
\hline \multicolumn{8}{|c|}{ SNP } \\
\hline 482 & & 58 & -1.133 & .2573 & 32 & -1.462 & .1437 \\
\hline 485 & & 52 & -1.811 & .0701 & 31 & -2.072 & .0383 \\
\hline 479 & & 59 & -1.316 & .1882 & 33 & -1.623 & .1045 \\
\hline 488 & & 55 & -1.065 & .2870 & 31 & -1.401 & .1611 \\
\hline 489 & & 59 & -1.316 & .1882 & 33 & -1.623 & .1045 \\
\hline \multicolumn{8}{|c|}{ Haplotype 482-485-479-488-489 } \\
\hline GATTT & .608 & 28 & -1.721 & .0853 & 31 & -1.994 & .0461 \\
\hline ATCCC & .381 & 26 & 1.453 & .1463 & 29 & 1.761 & .0782 \\
\hline \multicolumn{8}{|c|}{ DISC1 (Block 2) } \\
\hline \multicolumn{8}{|c|}{ SNP } \\
\hline 517 & & 28 & -3.215 & .0013 & 26 & -2.786 & .0053 \\
\hline 518 & & 39 & -2.694 & .0071 & 37 & -2.67 & .0076 \\
\hline \multicolumn{8}{|c|}{ Haplotype 517-518 } \\
\hline AG & .505 & 33 & -2.608 & .0091 & 31 & -2.177 & .0295 \\
\hline GA & .416 & 31 & 3.347 & .0008 & 30 & 3.146 & .0017 \\
\hline
\end{tabular}

HF, haplotype frequency.

${ }^{a} \mathrm{~N}$ : the number of the informative family.

${ }^{b} Z$ : the test statistic.

-105.14, AIC $=222.28)$, and the second haplotype block was linked and associated with the undegraded CPT d' (log likelihood $=-164.57$, AIC $=343.14$ ).

To further examine the relationship between the SNPs and CPT performance, we next treated schizophrenic patients' CPT performance as a covariate in the association between schizophrenia diagnosis and the SNPs. We first inferred each individual's haplotype using the SimWalk2 and then subjected the individual's clinical diagnosis (schizophrenic or not) to logistic regression on the haplotypes with the deficit status in CPT d' (with an adjusted $z$ score $\leq-2.5$ ) as a covariate using the PROC GENMOD. The reason for choosing -2.5 was based on a previous finding that a recurrence risk ratio analysis of the CPT among the nonpsychotic relatives of schizophrenic patients indicated that a criterion more stringent than 2 SDs below the population mean led to a risk ratio higher than schizophrenia itself (Chen et al 2004).
Because the effect of CPT d' was significant in these logistic regression analyses, we then stratified the sample into those without CPT deficit and those with CPT deficit, and conducted the logistic regression analysis separately for these two subgroups. It turned out that the haplotypes of the second block (i.e., SNP517-518) tended to exhibit signification association with schizophrenia in those with CPT deficit but not in those without CPT deficit (Table 3). For patients with a deficit on the undegraded CPT, a single copy of the haplotype GA had an increased risk for schizophrenia (those with genotype GA/GA or $\mathrm{GA} /$ others had an odds ratio [OR] of 3.84 or 3.86, respectively, in the narrow model and 5.48 or 5.72 , respectively, in the broad model), whereas two copies of haplotype AG had a protective effect for schizophrenia (those with genotype AG/AG had an OR of .19 in the narrow model and .13 in the broad model). However, for patients without deficit on the undegraded CPT, the pattern was less consistent (only those

Table 3. Logistic Regression Analysis of Schizophrenia on DISC1 Genetic Haplotypes of SNP517-SNP518 Stratified by CPT Deficit Status

\begin{tabular}{|c|c|c|c|c|c|c|c|c|c|c|c|}
\hline \multirow[b]{2}{*}{ CPT Version } & \multirow[b]{2}{*}{ Haplotypes } & \multicolumn{5}{|c|}{ Without CPT Deficit ${ }^{a}$} & \multicolumn{5}{|c|}{ With CPT Deficit } \\
\hline & & $N$ & OR & $p$ Value & OR & $p$ Value & $N$ & OR & $p$ Value & OR & $p$ Value \\
\hline \multirow[t]{3}{*}{ Undegraded CPT } & GA/GA & 20 & 1.67 & .342 & 1.04 & .941 & 15 & 3.84 & .074 & 5.48 & .003 \\
\hline & GA/others & 50 & .51 & .123 & .38 & .016 & 26 & 3.86 & .047 & 5.72 & .001 \\
\hline & Others/others & 40 & 1.00 & & 1.00 & & 17 & 1.00 & & 1.00 & \\
\hline & Others/others & 39 & 1.00 & & 1.00 & & 22 & 1.00 & & 1.00 & \\
\hline \multirow[t]{3}{*}{ Undegraded CPT } & AG/AG & 33 & .78 & .595 & 1.44 & .426 & 17 & .19 & .038 & 0.13 & .001 \\
\hline & AG/others & 54 & .83 & .647 & .97 & .931 & 23 & .63 & .562 & 0.58 & .398 \\
\hline & Others/others & 27 & 1.00 & & 1.00 & & 18 & 1.00 & & 1.00 & \\
\hline \multirow[t]{2}{*}{ Degraded CPT } & AG/AG & 28 & .38 & .066 & .52 & .156 & 22 & .66 & .371 & 1.18 & .814 \\
\hline & AG/others & 56 & .75 & .461 & .81 & .594 & 20 & .77 & .684 & 1.07 & .913 \\
\hline
\end{tabular}

CPT, Continuous Performance Test; OR, odds ratio.

${ }^{a} \mathrm{CPT}$ deficit was defined as an adjusted $z$ score of $\leq-2.5$. 
with genotype GA/others had a decreased risk for schizophrenia). Intriguingly, no association between SNP517-518 haplotypes and schizophrenia were found when the sample was stratified by the degraded CPT. When similar stratified analyses were conducted for the haplotypes of the first block (i.e., SNP482, 485, 479, 488, and 489), none of the haplotypes exhibited a significant association with schizophrenia in either those with or without CPT deficit (data not shown).

\section{Discussion}

We found two haplotype blocks using SNP fine mapping study around the D1S251 marker with significant association with schizophrenia. The first block of rs487047-rs508908-rs538643rs539699-rs578945 covers the genetic region of the GNPAT (or DHAPAT) gene, and the second block of rs2793092-rs2793091 is located within the DISC1 gene. The first block in the GNPAT gene encodes the dihydroxyacetone-phosphate acyltransferase enzyme (DHAPAT) that is located within peroxisomes and catalyzes the biosynthesis of ether phospholipids. This enzyme was found to be in deficiency in varying degrees in patients with congenital peroxisomal disorders, compared with normal controls (Hajra 1997; Schutgens et al 1984). A girl with deficient DHAPAT was reported to have severe mental retardation, developmental delay, and growth failure (Elias et al 1998). Our result suggests that GNPAT may also be involved in the broad definition of schizophrenia $(p=.046)$, although it may not play a major role because of its weak association. Since this is the first report of the potential involvement of the GNPAT gene in schizophrenia, further work examining the SNP markers located within the exons of the gene is warranted.

The second block that was significantly associated with schizophrenia is located within the DISC1 gene, and consistently exhibited significant associations in both single-locus and haplotype-based association analysis. The DISC1 gene was found to be closer to the peak NPL score of the D1S251 marker than to the adjacent lowest NPL score of the D1S404 marker in the previous Taiwan study (Hwu et al 2003). Furthermore, the D1S251 marker also showed a highest log of the odds (LOD) score of 2.5 ( $p=$ .002 ) in the populations of Britain and Iceland (Curtis et al 2003). Thus, this study in an expanded sample of affected sibpair families for $48 \%$ provides a replication of the linkage of DISC1 to schizophrenia. Although an inconsistent result has been reported in the Chinese ethnic group (Chen et al 2006), further analyses on the risk polymorphisms deserve further study.

In terms of haplotype analysis, our results suggest that the haplotypes consisting of SNPs in introns 4 and 5 of the DISC1 gene were significantly associated with schizophrenia. In contrast, the significant haplotype constituted by two SNPs for the Finnish population is located between the intron 1 and exon 2 (Hennah et al 2003). The discrepancy in the composition of the associated haplotype between the two studies could have two explanations. First, these reported regions may be in linkage disequilibrium, and the associations of the haplotypes merely suggest that the DISC1 gene is nearby a true susceptibility gene for schizophrenia. This possibility is further supported by the variations in linkage disequilibrium across ethnic groups. Second, both regions may be truly involved in the genetic susceptibility to schizophrenia. However, both regions of the DISC1 gene may interact with different cytoskeletal proteins. The exons 1 and 2 of the DISC1 gene encode the putative globular domains that bind strongly with the cytoplasmic microtubules of $\alpha$-tubulin, while the exons 3-13 encode the putative helical tail (coiled-coil motif) that is essential for interaction with neurodevelopmental protein NUDEL (Brandon et al 2004; Ozeki et al 2003). The exon 5, which covers the amino acid 425-467 of DISC1, may bind MIPT3 (Morris et al 2003), kendrin (Miyoshi et al 2004), and FEZ1 (Miyoshi et al 2003), and involve a self-associated domain that is related to the microtubule organization at the centrosome (Kamiya et al 2005). It is less likely to bind PDE4B, which requires amino acid 219-283 of DISC1 (Millar et al 2005; Sawa and Snyder 2005). If the second explanation holds, it implies different pathological pathways in different ethnic groups. Further investigation to see whether there are risk mutations in the exon 5 of DISC1 in our study population is warranted to distinguish these two possibilities.

There are two more differences in the results between the Finnish study (Hennah et al 2003) and ours. Neither the gender difference in the undertransmission of the DISC1 gene nor the association of the TRAX gene located upstream of the DISC1 gene with schizophrenia in the Finnish sample was found in our study. Nevertheless, the two studies did have a consistent finding that the DISC1 gene was not associated with the age onset of schizophrenia. Since we have ruled out the possible existence of the balanced translocation (q42.1; q14.3) in our schizophrenia DNA samples (Liu et al, unpublished results), the involvement of the DISC1 gene in our schizophrenic patients may be exerted through mechanisms other than genomic disruption.

One unique feature of this study is that we further revealed that the association of DISC1 with schizophrenia was limited to those with CPT deficits. The CPT is a measure of sustained attention and has been demonstrated to be highly sensitive to brain damage or dysfunction (Riccio et al 2002). Previous studies in Taiwanese population indicated that the heritability for the CPT performance in the nonpsychotic first-degree relatives of schizophrenic patients ranged from .48 to .62 (Chen et al 1998a), and the CPT deficits in schizophrenic patients were not amenable to 3-month neuroleptic treatment (Chen and Faraone 2000). In this study, the heritability for the CPT in the families of the affected sibpairs was even higher (.751 to .831). These consistent results indicate that the CPT deficits are likely to be a useful indicator for the genetic susceptibility to schizophrenia.

Under the AIC test for the optimum genetic model, the GNPAT genetic haplotype was significantly associated with the degraded CPT scores, whereas the DISC1 genetic haplotype was significantly associated with the undegraded CPT scores. After further stratifying the CPT performance into deficit or nondeficit according to the threshold of an adjusted $z$ score of -2.5 , we found that the GA haplotype of the DISC1 gene was found to be the dominant susceptible indicator for the undegraded CPT deficit, yet the AG haplotype was the dominant protective indicators for the CPT deficit. In contrast, GNPAT did not show such an association with schizophrenia in the similar stratification analysis. The differential association of both versions of the CPT with the two blocks might be due to chance finding, since the significance level of some association was borderline only (e.g., the GNPAT block with the degraded CPT). Another possibility is that the two versions of the CPT tap different aspects of neuropsychological functioning: the undegraded session involved mainly a working memory component, whereas the degraded session included a sensory-perceptual component as well (Chen and Faraone 2000). Our results suggest that the DISC1 gene, especially the intron 4 -intron 5 region, may be involved in regulating sustained attention per se (i.e., working memory) of schizophrenia. The opposite direction in predicting the risk of 
sustained attention deficit for the GA and AG haplotypes might be accounted for by binding affinities with different regulators. Intriguingly, recent studies using different neuropsychological instruments also reported similar association of the DISC1 gene with memory-related neuropsychological impairment in schizophrenia, such as a haplotype at exon 8 with impaired semantic processing in long-term memory (Cannon et al 2005), a haplotype between exon 1 and exon 2 with poorer short-term visual memory and attention (Hennah et al 2005), SNP hCV1650649 with rapid search and verbal working memory (Burdick et al 2005), as well as exon 11 variation (rs821616) with cognitive ageing (Thomson et al 2005). Whether all these findings point to a specific neuropsychological mechanism that is regulated via the DISC1 gene warrants further investigation.

The DISC1 locus at 1q42 receives linkage support not only in schizophrenia but also in schizoaffective disorder (Hamshere et al 2005) and bipolar disorder (Macgregor et al 2004). Evidence for allelic association of polymorphisms at this locus has been reported not only for schizophrenia, but also for schizoaffective disorder and bipolar disorder (Hodgkinson et al 2004; Thomson et al 2005). Recently, the DISC1 has even been proposed as a common susceptibility gene for both schizophrenia and mood disorder (Craddock et al 2006). In this study, we found that the DISC1 locus was specifically associated with the endophenotype of CPT in schizophrenia. The CPT deficits in schizophrenic inpatients persisted from admission to discharge despite improvements in clinical symptoms (Cornblatt et al 1997; Epstein et al 1996; Liu et al 2002). However, the CPT deficit improved in bipolar disorder when remission was achieved (Liu et al 2002; Sax et al 1998; Sax et al 1999). Thus, the CPT deficit is a stable vulnerability indicator for schizophrenia but a mediator for bipolar disorder (Liu et al 2002). If DISC1 is involved in the pathophysiologic process of the two disorders through CPT deficits, we suggest that it may play a vulnerability role in schizophrenia but a modifying role in bipolar disorder.

Unlike the results of the CPT, the WCST scores were associated with neither the DISC1 nor the GNPAT gene. It appears that the 1q42.1 region may have little contribution to the pathologic process of executive functioning in schizophrenia.

In summary, a 2-cM region surrounding the D1S251 marker was screened with SNPs for genetic association with schizophrenia. Two blocks of SNP haplotypes in this region showed significant associations with schizophrenia. These two blocks were located within the regions of GNPAT (a five-SNP block) and DISC1 (a two-SNP block) genes, respectively. The potential involvement of GNPAT with schizophrenia is preliminary, while the finding for DISC1 is a replication of previous studies. We further revealed that the association of DISC1 with schizophrenia was limited to those with CPT deficits and may indicate a potential biological pathway for future investigation.

This study was supported by the grants from National Research Program for Genomic Medicine, National Science Council, Taiwan (NSC-91-3112-B-002-011; NSC-92-3112-B-002-019; NSC-93-3112B-002-012; NSC-94-3112-B-002; DOH94-TD-G-111-035), from National Health Research Institute, Taiwan (NHRI-90-8825PP; NHRI-EX91, 92, 93-9113PP,) and from National Institute of Mental Health (NIMH), Bethesda, MD (IRO1 MH59624-01).

We acknowledge the SNP genotyping work done by the National Genotyping Center (NGC), NSC, Taiwan, and the belp from the department of Medical Research, National Taiwan University Hospital, Taipei, Taiwan.
Abecasis GR, Cardon LR, Cookson WO (2000a): A general test of association for quantitative traits in nuclear families. Am J Hum Genet 66:279-292.

Abecasis GR, Cherny SS, Cookson WO, Cardon LR (2002): Merlin-rapid analysis of dense genetic maps using sparse gene flow trees. Nat Genet 30:97-101.

Abecasis GR, Cookson WO, Cardon LR (2000b): Pedigree tests of transmission disequilibrium. Eur J Hum Genet 8:545-551.

Andreasen NC (1983): The Scale for the Assessment of Negative Symptoms (SANS). lowa City, IA: University of lowa.

Andreasen NC (1984): The Scale for the Assessment of Positive Symptoms (SAPS). lowa City, IA: University of lowa.

Barrett JC, Fry B, Maller J, Daly MJ (2005): Haploview: Analysis and visualization of LD and haplotype maps. Bioinformatics 21:263-265.

Bassett AS, Chow EW, Vieland VJ, Brzustowicz L (2002): Is schizophrenia linked to chromosome 1q? Science 298:2277; author reply 2277.

Blackwood DH, Fordyce A, Walker MT, St Clair DM, Porteous DJ, Muir WJ (2001): Schizophrenia and affective disorders-cosegregation with a translocation at chromosome 1q42 that directly disrupts brain-expressed genes: Clinical and P300 findings in a family. Am J Hum Genet 69:428-433.

Brandon NJ, Handford EJ, Schurov I, Rain JC, Pelling M, Duran-Jimeniz B, et al (2004): Disrupted in Schizophrenia 1 and Nudel form a neurodevelopmentally regulated protein complex: Implications for schizophrenia and other major neurological disorders. Mol Cell Neurosci 25:42-55.

Burdick KE, Hodgkinson CA, Szeszko PR, Lencz T, Ekholm JM, Kane JM, et al (2005): DISC1 and neurocognitive function in schizophrenia. Neuroreport 16:1399-1402.

Callicott JH, Straub RE, Pezawas L, Egan MF, Mattay VS, Hariri AR, et al (2005): Variation in DISC1 affects hippocampal structure and function and increases risk for schizophrenia. Proc Natl Acad Sci U S A 102:8627-8632.

Cannon TD, Hennah W, van Erp TG, Thompson PM, Lonnqvist J, Huttunen M, et al (2005): Association of DISC1/TRAX haplotypes with schizophrenia, reduced prefrontal gray matter, and impaired short- and long-term memory. Arch Gen Psychiatry 62:1205-1213.

Chen QY, Chen Q, Feng GY, Lindpaintner K, Wang LJ, Chen ZX, et al (in press): Case-control association study of Disrupted-in-Schizophrenia-1 (DISC1) gene and schizophrenia in the Chinese population. J Psychiatr Res.

Chen WJ, Chang C-H, Liu SK, Hwang TJ, Hwu H-G, Collaborators from the Multidimensional Psychopathology Group Research Project (2004): Sustained attention deficits in nonpsychotic relatives of schizophrenic patients: A recurrence risk ratio analysis. Biol Psychiatry 55:995-1000.

Chen WJ, Faraone SV (2000): Sustained attention deficits as markers of genetic susceptibility to schizophrenia. Am J Med Genet (Seminars in Medical Genetics) 97:52-57.

Chen WJ, Hsiao CK, Hsiao L-L, Hwu H-G (1998a): Performance of the Continuous Performance Test among community samples. Schizophr Bull 24: 163-174.

Chen WJ, Liu SK, Chang CJ, Lien YJ, Chang YH, Hwu HG (1998b): Sustained attention deficit and schizotypal personality features in nonpsychotic relatives of schizophrenic patients. Am J Psychiatry 155:1214-1220.

Clayton D (1999): A generalization of the transmission/disequilibrium test for uncertain-haplotype transmission. Am J Hum Genet 65:1170-1177.

Cornblatt B, Obuchowski M, Schnur DB, O'Brien JD (1997): Attention and clinical symptoms in schizophrenia. Psychiatr Q 68:343-359.

Cornblatt BA, Keilp JG (1994): Impaired attention, genetics, and the pathophysiology of schizophrenia. Schizophr Bull 20:31-46.

Craddock N, O'Donovan MC, Owen MJ (2006): Genes for schizophrenia and bipolar disorder? Implications for psychiatric nosology. Schizophr Bull 32:9-16.

Curtis D, Kalsi G, Brynjolfsson J, Mclnnis M, O'Neill J, Smyth C, et al (2003): Genome scan of pedigrees multiply affected with bipolar disorder provides further support for the presence of a susceptibility locus on chromosome 12q23-q24, and suggests the presence of additional loci on $1 p$ and 1q. Psychiatr Genet 13:77-84.

Elias ER, Mobassaleh M, Hajra AK, Moser AB (1998): Developmental delay and growth failure caused by a peroxisomal disorder, dihydroxyacetonephosphate acyltransferase (DHAP-AT) deficiency. Am J Med Genet 80: 223-226.

Epstein Jl, Keefe RS, Roitman SL, Harvey PD, Mohs RC (1996): Impact of neuroleptic medications on continuous performance test measures in schizophrenia. Biol Psychiatry 39:902-905.

Goldberg TE, Weinberger DR, Berman KF, Pliskin NH, Podd MH (1987): Further evidence for dementia of the prefrontal type in schizophrenia? A 
controlled study of teaching the Wisconsin Card Sorting Test. Arch Gen Psychiatry 44:1008-1014.

Gottesman II, Gould TD (2003): The endophenotype concept in psychiatry: Etymology and strategic intentions. Am J Psychiatry 160:636-645.

Hajra AK (1997): Dihydroxyacetone phosphate acyltransferase. Biochim Biophys Acta 1348:27-34.

Hamshere ML, Bennett P, Williams N, Segurado R, Cardno A, Norton N, et al (2005): Genomewide linkage scan in schizoaffective disorder: significant evidence for linkage at 1q42 close to DISC1, and suggestive evidence at 22q11 and 19p13. Arch Gen Psychiatry 62:1081-1088.

Heaton RK, Chelune Gl, Talley JL, Kay GG, Curtiss G (1993): Wisconsin Card Sorting Test Manual: Revised and Expanded. Odessa, FL: Psychological Assessment Resources.

Hedrick PW (1987): Gametic disequilibrium measures: proceed with caution. Genetics 117:331-341

Hennah W, Tuulio-Henriksson A, Paunio T, Ekelund J, Varilo T, Partonen T, et al (2005): A haplotype within the DISC1 gene is associated with visual memory functions in families with a high density of schizophrenia. Mol Psychiatry 10:1097-1103.

Hennah W, Varilo T, Kestila M, Paunio T, Arajarvi R, Haukka J, et al (2003): Haplotype transmission analysis provides evidence of association for DISC1 to schizophrenia and suggests sex-dependent effects. Hum Mol Genet 12:3151-3159.

Hodgkinson CA, Goldman D, Jaeger J, Persaud S, Kane JM, Lipsky RH, Malhotra AK (2004): Disrupted in schizophrenia 1 (DISC1): Association with schizophrenia, schizoaffective disorder, and bipolar disorder. Am J Hum Genet 75:862-872.

Horvath S, Xu X, Laird NM (2001): The family based association test method: Strategies for studying general genotype-phenotype associations. Eur J Hum Genet 9:301-306.

Horvath S, Xu X, Lake SL, Silverman EK, Weiss ST, Laird NM (2004): Familybased tests for associating haplotypes with general phenotype data: Application to asthma genetics. Genet Epidemiol 26:61-69.

Hovatta I, Varilo T, Suvisaari J, Terwilliger JD, Ollikainen V, Arajarvi R, et al (1999): A genomewide screen for schizophrenia genes in an isolated Finnish subpopulation, suggesting multiple susceptibility loci. Am J Hum Genet 65:1114-1124.

Hwu HG (1999): Psychiatric Diagnostic Assessment. Taipei: Publication Committee, Medical College, National Taiwan University.

Hwu HG, Liu CM, Fann CS, Ou-Yang WC, Lee SF (2003): Linkage of schizophrenia with chromosome $1 \mathrm{q}$ loci in Taiwanese families. Mol Psychiatry $8: 445-452$.

Kamiya A, Kubo K, Tomoda T, Takaki M, Youn R, Ozeki Y, et al (2005): A schizophrenia-associated mutation of DISC1 perturbs cerebral cortex development. Nat Cell Biol 7:1167-1178.

Koren D, Seidman LJ, Harrison RH, Lyons MJ, Kremen WS, Caplan B, et al (1998): Factor structure of the Wisconsin Card Sorting Test: Dimensions of deficit in schizophrenia. Neuropsychology 12:289-302.

Laird NM, Horvath S, Xu X (2000): Implementing a unified approach to familybased tests of association. Genetic Epidemiology 19(Suppl 1):S36-42.

Levinson DF, Holmans PA, Laurent C, Riley B, Pulver AE, Gejman PV, et al (2002): No major schizophrenia locus detected on chromosome 1q in a large multicenter sample. Science 296:739-741.

Lin CCH, Chen WJ, Yang H-J, Hsiao CK, Tien AY (2000): Performance on the Wisconsin Card Sorting Test among adolescents in Taiwan: Norms, factorial structure, and relation to schizotypy. J Clin Exp Neuropsychol 22: 69-79.

Liu SK, Chiu CH, Chang CJ, Hwang TJ, Hwu HG, Chen WJ (2002): Deficits in sustained attention in schizophrenia and affective disorders: stable versus state-dependent markers. Am J Psychiatry 159:975-982.

Macgregor S, Visscher PM, Knott S, Porteous D, Muir W, Millar K, Blackwood D (2002): Is schizophrenia linked to chromosome 1q? Science 298:2277; author reply 2277.

Macgregor S, Visscher PM, Knott SA, Thomson P, Porteous DJ, Millar JK, et al (2004): A genome scan and follow-up study identify a bipolar disorder susceptibility locus on chromosome 1q42. Mol Psychiatry 9:1083-1090.

Millar JK, Christie S, Anderson S, Lawson D, Hsiao-Wei Loh D, et al (2001): Genomic structure and localisation within a linkage hotspot of disrupted in schizophrenia 1, a gene disrupted by a translocation segregating with schizophrenia. Mol Psychiatry 6:173-178.

Millar JK, Pickard BS, Mackie S, James R, Christie S, Buchanan SR, et al (2005): DISC1 and PDE4B are interacting genetic factors in schizophrenia that regulate CAMP signaling. Science 310:1187-1191.
Millar JK, Wilson-Annan JC, Anderson S, Christie S, Taylor MS, Semple CA, et al (2000): Disruption of two novel genes by a translocation co-segregating with schizophrenia. Hum Mol Genet 9:1415-1423.

Miyoshi K, Asanuma M, Miyazaki I, Diaz-Corrales FJ, Katayama T, Tohyama M, Ogawa N (2004): DISC1 localizes to the centrosome by binding to kendrin. Biochem Biophys Res Commun 317:1195-1199.

Miyoshi K, Honda A, Baba K, Taniguchi M, Oono K, Fujita T, et al (2003): Disrupted-In-Schizophrenia 1, a candidate gene for schizophrenia, participates in neurite outgrowth. Mol Psychiatry 8:685-694.

Morris JA, Kandpal G, Ma L, Austin CP (2003): DISC1 (Disrupted-In-Schizophrenia 1) is a centrosome-associated protein that interacts with MAP1A, MIPT3, ATF4/5 and NUDEL: Regulation and loss of interaction with mutation. Hum Mol Genet 12:1591-1608.

Nuechterlein KH (1991): Vigilance in Schizophrenia and Related Disorders. Amsterdam: Elsevier.

O'Connell JR, Weeks DE (1998): PedCheck: A program for identification of genotype incompatibilities in linkage analysis. Am J Hum Genet 63:259266.

Ozeki Y, Tomoda T, Kleiderlein J, Kamiya A, Bord L, Fujii K, et al (2003): Disrupted-in-Schizophrenia-1 (DISC-1): Mutant truncation prevents binding to NudE-like (NUDEL) and inhibits neurite outgrowth. Proc Natl Acad Sci U S A 100:289-294.

Riccio CA, Reynolds CR, Lowe P, Moore JJ (2002): The continuous performance test: A window on the neural substrates for attention? Arch Clin Neuropsychol 17:235-272.

Risch N (1990): Linkage strategies for genetically complex traits. I. Multilocus models. Am J Hum Genet 46:222-228.

Robinson AL, Heaton RK, Lehman RA, Stilson DW (1980): The utility of the Wisconsin Card Sorting Test in detecting and localizing frontal lobe lesions. J Consult Clin Psychol 48:605-614.

Rodi CP, Darnhofer-Patel B, Stanssens P, Zabeau M, van den Boom D (2002): A strategy for the rapid discovery of disease markers using the MassARRAY system. Biotechniques June(Suppl):62-6, 68-9.

Rosvold HE, Mirsky AF, Sarason I, Bransome ED, Jr., Beck LH (1956): A Continuous Performance Test of brain damage. J Consult Psychol 20:343-350.

Sawa A, Snyder SH (2005): Genetics. Two genes link two distinct psychoses. Science 310:1128-1129.

Sax KW, Strakowski SM, Keck PE Jr, McElroy SL, West SA, Stanton SP (1998): Symptom correlates of attentional improvement following hospitalization for a first episode of affective psychosis. Biol Psychiatry 44:784-786.

Sax KW, Strakowski SM, Zimmerman ME, DelBello MP, Keck PE Jr, Hawkins JM (1999): Frontosubcortical neuroanatomy and the continuous performance test in mania. Am J Psychiatry 156:139-141.

Schutgens RB, Romeyn GJ, Wanders RJ, van den Bosch H, Schrakamp G, Heymans HS (1984): Deficiency of acyl-CoA: Dihydroxyacetone phosphate acyltransferase in patients with Zellweger (cerebro-hepato-renal) syndrome. Biochem Biophys Res Commun 120:179-184.

Sobel E, Lange K (1996): Descent graphs in pedigree analysis: Applications to haplotyping, location scores, and marker-sharing statistics. Am J Hum Genet 58:1323-1337.

Sobel E, Papp JC, Lange K (2002): Detection and integration of genotyping errors in statistical genetics. Am J Hum Genet 70:496-508.

Sobel E, Sengul H, Weeks DE (2001): Multipoint estimation of identity-bydescent probabilities at arbitrary positions among marker loci on general pedigrees. Hum Hered 52:121-131.

Terwilliger JD, Ott J (1994): Handbook of Human Genetic Linkage. Baltimore: Johns Hopkins University Press.

Thomson PA, Wray NR, Millar JK, Evans KL, Hellard SL, Condie A, et al (2005): Association between the TRAX/DISC locus and both bipolar disorder and schizophrenia in the Scottish population. Mol Psychiatry 10:657$658,616$.

Tien AY, Spevack TV, Jones DW, Pearlson GD, Schlaepfer TE, Strauss ME (1996): Computerized Wisconsin Card Sorting Test: comparison with manual administration. Kaohsiung J Med Science 12:479-85.

Tost J, Gut IG (2005): Genotyping single nucleotide polymorphisms by MALD mass spectrometry in clinical applications. Clin Biochem 38:335-50.

Weinberger DR, Berman KF, Illowsky BP (1988): Physiological dysfunction of dorsolateral prefrontal cortex in schizophrenia. III. A new cohort and evidence for a monoaminergic mechanism. Arch Gen Psychiatry 45:609-15.

Wolf LE, Cornblatt BA, Roberts SA, Shapiro BM, Erlenmeyer-Kimling L (2002): Wisconsin Card Sorting deficits in the offspring of schizophrenics in the New York High-Risk Project. Schizophr Res 57:173. 